\title{
Gender differences in injuries among rural youth
}

\author{
Cheryl S Alexander, Mark R Somerfield, Margaret E Ensminger, Young J Kim, Karin E \\ Johnson
}

\begin{abstract}
Goal-This paper presents injury data from the first year of a three year longitudinal study of risk taking behaviors among adolescents.

Sample-Study subjects were a cohort of 758 rural students from Maryland's Eastern Shore who were in the eighth grade in 1987.
\end{abstract}

Methods-Students completed a 45 minute, self administered survey in which they reported numbers of injuries experienced in the past year, risk taking behaviors, anger expression, delinquency, alcohol and drug use, physical exercise, work experience, and level of parental supervision. In addition, students had their height and weight measurements taken by trained research staff and completed a self rating of pubertal development using Tanner drawings.

Results-Slightly more than half $(53 \cdot 2 \%)$ of the boys and over one third $(37 \cdot 7 \%)$ of the girls reported experiencing one or more medically attended injuries during the last year. Poisson regression analyses were conducted to estimate the extent to which gender differences in injuries could be accounted for by adolescent behaviors. Gender effects became non-significant when adjustments were made for risk taking, school discipline problems, and exercise frequency. Gender differences in injuries were reduced but remained significant when substance use, employment, and anger were controlled. Poisson regression analyses were conducted separately for males and females to assess whether factors associated with injuries were similar across genders. For boys, risk taking, anger, and school discipline problems were significantly related to number of injuries. Boys with a low body mass index and late pubertal development (mean ratio 3.09 ), as well as those with high body mass index and early pubertal development (mean ratio $2 \cdot 16$ ), reported greater numbers of injuries than average boys. For girls, substance use, cruising, risk taking, anger, and exercise frequency were significantly associated with injuries. Girls with an early onset of menses reported, on average, twice the number of injuries than those who were on time. Girls with high body mass index who were late in their pubertal development reported, on average, five times more injuries than other girls.
Conclusions-Although gender is a significant risk factor for injuries, certain behaviors like risk taking, school related delinquency, and physical exercise partially explain the higher number of injuries among adolescent males in this study. For both males and females, indicators of pubertal and physical development are important factors to consider in studies of injuries during early adolescence.

(Injury Prevention 1995; 1: 15-20)

Keywords: rural populations, pubertal development, risk taking behaviors, gender.

Injuries kill or disable more adolescents than any other disease or condition. They account for $57 \%$ of deaths among those $15-19$ years of age. ${ }^{1}$ In addition to being the major cause of mortality for adolescents, many young people incur crippling disabilities as a result of injuries. Estimates derived from Massachusetts data indicate that, for every injury related death of an adolescent, there may be as many as 41 hospitalizations, and over 1000 emergency room visits. ${ }^{2}$

Being male has consistently been identified as a significant risk factor for injuries. ${ }^{3-7}$ However, researchers have suggested that it is not the biological characteristic of being male that is related to injuries, but rather the behaviors associated with the male sex role that increase injury risk. ${ }^{4}$

There are several ways in which behavior may account for gender differences in adolescent injuries. First, there may be differences in the types of behaviors that males and females engage in that are injury related. These include problem behaviors, ${ }^{89}$ risk taking, ${ }^{10}$ alcohol and drug use, ${ }^{112}$ sports, ${ }^{13}$ and employment. ${ }^{14}$ Second, males and females may differ in the frequency with which they engage in a risk behavior, thus resulting in differential exposure to injury. Third, the contexts in which behaviors occur may be gender related. For example, males may be more likely than females to drive between 9:00 pm and 6:00 am when the greatest number of motor vehicle injuries occur. ${ }^{15}$

In this paper we examine the extent to which gender differences in injuries among young adolescents can be explained by their behaviors. In addition, we identify those behaviors that are associated with injuries for adolescent males and females, separately. By understanding more about behaviors that predispose adolescent injury, we can plan more effective preventive interventions. 


\section{Subjects and methods}

POPULATION

Data for this paper come from the first year of a three year longitudinal cohort study (1987-9) of adolescents from three rural counties in Maryland. The study received Institutional Review Board approval from Johns Hopkins University and a Certificate of Confidentiality was secured from the National Institute of Drug Abuse. In 1986, active parental consent for study participation was sought for all eighth grade students in the public schools of the three counties. Of the eighth graders eligible, 758 , or $64 \%$, were enrolled in the study. Objections to questions on sexual behavior seemed to have influenced some parents to withhold consent for study participation. To determine whether the students who participated in the study differed from non-participants, comparisons were made for race and gender, the only two variables for which information on nonparticipants was made available to the study by the schools. No significant differences were found in these comparisons. It is likely, based on our experience with school personnel and school boards, that parents who refused study participation held more politically and religiously conservative views than those parents who allowed their children to be study subjects.

\section{METHODS}

Students completed a self administered questionnaire in the spring of eighth grade during a 45 minute class period. At a separate assessment, they had height and weight measurements taken by trained research staff and were asked to self rate their pubertal development using Tanner drawings ${ }^{16}$ and a brief questionnaire.

\section{MEASURES}

Injuries were assessed by asking students to report the number experienced in the past year for which they had received treatment by a physician or a nurse.

The following variables were selected as predictors of injury based on previous research. Most can be grouped into one or two broad categories: healthy behaviors and health compromising or problem behaviors.

\section{Healthy behaviors}

Physical activity-This variable was measured by asking students how many hours per week they spent exercising, not counting physical education classes. The number of hours ranged from 0-10 hours.

Labor force participation-Students were asked to report the number of hours per week they worked outside of the home for pay. Time ranged from 0 to 20 or more hours per week.

Health compromising behaviors Substance use-Two indicators of substance use were included: alcohol use in the past 30 days and lifetime marijuana use. (A lifetime measure was selected due to the low frequency of marijuana use reported within the past 30 days.) Responses for alcohol use were categorized as 0 days, $1-2$ days, and 3 or more days per month; for marijuana as 0 times, $1-5$ times, and 6 or more times ever used.

Risk taking-Two measures of risk taking propensity were assessed. The Adolescent Risk Taking Scale, constructed for the study, was used to assess the extent to which young people engage in physically daring feats and minor delinquent acts. ${ }^{17}$ This nine item, three point summated scale has a Cronbach's coefficient alpha of 0.78 indicating good internal consistency. Cruising, defined as riding around in cars with no particular destination or purpose in mind, served as an indicator of increased exposure to the opportunity for injury. Frequency of cruising was measured using five response categories from 'not at all' to 'several times per week'.

School discipline problems-Discipline problems at school were measured by asking students whether, since the beginning of eighth grade, they had been in enough trouble to be sent to the principal's office.

Anger-Expressions of anger were used to reflect aggressive behavior. A five item Likert scale developed for the Woodlawn study in Chicago measured ways of expressing anger including yelling, hitting, and fighting. ${ }^{18}$ Higher scores indicated more overt anger expressions. The Cronbach's alpha coefficient for this scale was 0.62 in eighth grade.

\section{Other variables}

In addition to the adolescent behaviors, we included a measure of parental supervision or monitoring, and two measures of physical development. Lack of parental supervision has been suggested as an antecedent of injury among children. ${ }^{19}$ Variations in physical and pubertal development may also contribute to injury risk through differential exposure to injury related activities such as sports participation.

Parental supervision-This four item scale measured the degree to which parents set rules for homework, curfew on weekdays and weekends, and the choice of friends and places where their adolescents are permitted to go. Cronbach's alpha coefficient for this scale was 0.68 .

Physical development-Two indicators of physical development were included. Each measure was computed separately for boys and girls. The body mass index (BMI), a ratio of weight to height squared, assessed body size. Pubertal development was measured by menarcheal status for girls and by pubic hair and penis development for boy. Tanner assessments ${ }^{16}$ were not used for girls due to limited variability in the ranges of scores for breast and pubic hair development: none of the girls self 
rated their breast or pubic hair development as a Tanner stage 1 and only three girls rated themselves as in stage 2. For eighth grade boys, there was greater variability in self assessed penis growth and pubic hair development. Scores ranged from 4 to 10 from a possible range of 2 to 10 . Boys with scores of 9 or 10 were defined are early maturers, boys with scores 7 or 8 were considered 'on-time', and those with scores 6 or below were late maturers. For girls, early maturation was defined as having the onset of menarche before age 11 , on-time as between 11 and 13 years, and late as older than 13 years of age.

Two measures of sociodemographic risk were also included: the race of the adolescent and the education of the parent or guardian who was interviewed when the adolescent was in ninth grade as part of the larger, three year study. Education of the interviewed parent or guardian served as a proxy for socioeconomic status.

\section{STATISTICAL METHODS}

Since our aim was to model the number of injuries as a function of adolescent behaviors, we used Poisson regression analyses. A Poisson model is appropriate for a count variable like number of injuries. It uses a log linear model where the logarithm of the outcome (that is, injuries) is regressed on the independent or predictor variables (for example, adolescent behaviors). ${ }^{20}$ In Poisson regression, the exponent (antilog) of the estimated regression gives a mean ratio. This is the relative estimated mean of the outcome variable associated with a category of the independent variable, relative to the reference category..$^{20}$ The mean ratio from the Poisson regression model is similar to the better known odds ratio from a logistic regression model, except, as in the case of injuries, the mean ratio refers to the estimated mean number of injuries while the odds ratio refers to the estimated odds of being injured relative to the reference group. For example, a gender coefficient of 1.50 (where males are coded ' 1 ' and females coded ' 0 ') would indicate that, on average, males experience $50 \%$ more injuries than females.

The Poisson regression analyses were conducted using the generalized linear model program (GLIM). A series of regression models were constructed to estimate the net effect of gender on the number of injuries controlling for adolescent behaviors, parental supervision, and sociodemographic characteristics. In the first model, numbers of injuries were regressed on gender, alone. In the next series of models, gender was retained in each model, and independent variables were added to and removed from the model, one at a time. The purpose of these analyses was to assess the effect of gender on injuries, controlling for the influence of other variables.

We were especially interested in observing which adolescent behaviors reduced gender effects on injury. Significant decreases in the magnitude of the gender coefficient in a given model indicated an attenuation of the relation- ship between gender and injuries as a function of adjustment for a second variable. For example, if the gender coefficient indicating a greater number of injuries for males as compared with females was significantly reduced when alcohol use was controlled, it would suggest that gender differences in eighth grade injuries can be explained in part by differences in drinking behaviors.

Second, Poisson regression analyses were conducted to estimate the effects of various adolescent behaviors, pubertal and physical development, and parental supervision on injuries for boys and girls separately. For these analysis, all independent variables entered the regression model simultaneously.

\section{Results}

INJURY PREVALENCE

Table 1 presents the distribution of injuries by gender. As expected, males reported a greater number of injuries than did females. Over half of the males reported one or more injuries as compared with $38 \%$ of females. Among students who were injured, the average numbers of injuries were fairly comparable for males and females.

\section{ADJUSTED GENDER EFFECTS ON INJURIES}

Next, we considered the extent to which gender differences in injuries seen in table 1 could be accounted for by adolescent behaviors. Overall, boys had on average $32 \%$ more injuries than girls. These gender effects became nonsignificant when adjustments were made for risk taking, school discipline problems, and exercise frequency (table 2). Gender differences in injuries were also reduced but still remained significant when substance use, employment, and anger were controlled.

\section{FACTORS RELATED TO GENDER SPECIFIC} INJURIES

To assess whether factors associated with injuries were similar for adolescent boys and girls, we examined the multivariate effects of adolescent behaviors, parent supervision, measures of physical development, and sociodemographic characteristics on the number of injuries separately by gender.

Measures of pubertal development and BMI were included in these analyses to account for variations in physical and pubertal develop-

\section{Table 1 Distribution of injuries by gender}

\begin{tabular}{lcc}
\hline & $\begin{array}{l}\text { Males } \\
(n=308)\end{array}$ & $\begin{array}{l}\text { Females } \\
(n=292)\end{array}$ \\
\hline $\begin{array}{l}\text { No of injuries } \\
\% \text { Of students injured }\end{array}$ & 371 & 276 \\
$\%$ Of students with injuries & $53 \cdot 2$ & $37 \cdot 7$ \\
0 & $46 \cdot 8$ & 62.3 \\
1 & $22 \cdot 4$ & 16.4 \\
2 & $16 \cdot 6$ & $8 \cdot 9$ \\
3 & $6 \cdot 8$ & $4 \cdot 1$ \\
$4+$ & $7 \cdot 4$ & $8 \cdot 3$ \\
Mean No of injuries among & $1 \cdot 20$ & 0.94 \\
all students & & \\
Mean No of injuries among & $2 \cdot 30$ & 2.50 \\
injured students & & \\
\hline
\end{tabular}


Table 2 Adjusted mean ratios for injuries by gender $(n=562)$

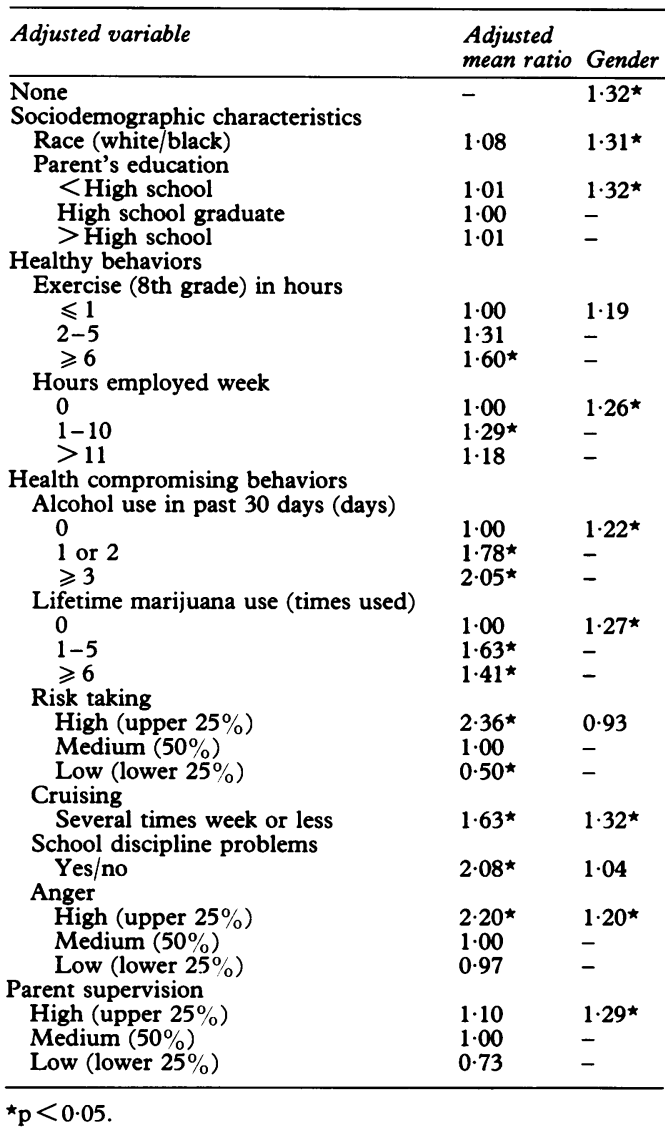

ment within gender groups. BMI was categorized into quartiles based on distributions for each gender with the middle $50 \%$ group as the reference category. BMI was categorized for ease in interpretation of findings because we were more interested in examining the effects of the extremes of body size (having a large body size (high on BMI) or a small body size (low on BMI)) on injuries, than incremental changes in body size associated with numbers of injuries.

Table 3 displays the results of the gender specific Poisson regression analyses. For boys, risk taking, anger, and school discipline problems were significantly related to number of injuries. The strongest association with injuries was for the interaction of physical developmental and pubertal development measures. Boys who were underdeveloped physically and sexually (mean ratio 3.09), as well as those who were highly physically and sexually developed (mean ratio $2 \cdot 16$ ), reported a greater number injuries than average boys. Other interactions, such as low pubertal development and high BMI, were not statistically significant.

Deviant behaviors, such as substance use, cruising, and risk taking, were significantly associated with injuries for girls. Girls who reported higher levels of anger also reported a greater number of injuries than their peers. In addition, girls who exercised more than six hours per week reported more injuries. There is evidence of physical and pubertal developmental effects on injury for girls as well: girls with early onset of menses reported on average twice the number of injuries than those who were on time. Girls with high body mass who were late in their pubertal development reported on average five times more injuries than other girls.

\section{Discussion}

Gender is a significant risk factor for adolescent injuries. In this study, eighth grade males reported more injuries than females. Findings suggest that certain behaviors that males engage in at least partially account for their higher risk for injuries. Our results show that by adjusting for risk taking, school discipline problems, and exercise, gender differences are greatly reduced.

Taking into account types of behaviors and their frequency of occurrence does not address gender differences in the manner in which behaviors are performed or the contexts in which they occur. For example, participation in team sports for girls may carry a different risk for injury than participation in team sports for boys because males and females do not typically play on the same teams or play the same sports. Similarly, knowing that a teenager drinks on three or more days per month does not say anything about how the person drinks, what kind of alcohol is drunk, the quantity consumed at a single drinking episode, or the circumstances under which the drinking takes place.

Our data did not permit an assessment of contextual influences on injury. Information about the nature of the risk behaviors and the contexts in which they occur might further reduce gender differentials observed for injuries. This type of information requires the use of more qualitative data collection methods, like process analysis. ${ }^{21}$ In process analysis, details surrounding the injury event, including for instance the immediate environment, social situation, and the individual's own internal cues, can be elicited through interviews. Detailed analyses of these data can separate out the antecedents of the event from its consequences, providing clues to gender related differences.

Findings from the analyses of gender specific injuries highlight the role of the meaning of biologic and social development in adolescent injuries. For example, pubertal development and body size were significantly associated with injuries in young adolescent boys and girls. Eighth graders are typically 13 years old. Because girls reach puberty on average two years before boys, there is greater variability in the size, shape, strength, and sexual maturity of eighth grade boys than of eighth grade girls. ${ }^{16}$ Such variation in development may have contributed to the increased injuries observed for two groups of eighth grade boys: those who were both highly physically and sexually developed and those who were underdeveloped. For boys, the social meaning of physical size may be very important in contributing to the risk of injury. Boys who are small for their age may be defensive about their size and may take unnecessary risks or pick fights to prove their 
Table 3 Poisson regression model of injuries for eighth grade males and females

\begin{tabular}{|c|c|c|c|c|c|c|}
\hline & \multicolumn{3}{|c|}{ Male $(n=252)$} & \multicolumn{3}{|c|}{ Female $(n=242)$} \\
\hline & Beta & $\begin{array}{l}\text { Mean } \\
\text { ratio }\end{array}$ & $\begin{array}{l}95 \% \\
\mathrm{CI} \\
\end{array}$ & Beta & $\begin{array}{l}\text { Mean } \\
\text { ratio }\end{array}$ & $\begin{array}{l}95 \% \\
C I^{\star}\end{array}$ \\
\hline $\begin{array}{l}\text { Intercept } \\
\text { Race (white/black) }\end{array}$ & $\begin{array}{r}-0.48 \\
0.28\end{array}$ & $\overline{1} \cdot 32$ & 0.978 to 1.738 & $\begin{array}{l}-1 \cdot 36 \\
-0 \cdot 16\end{array}$ & $\overline{0} \cdot 85$ & 0.667 to 1.229 \\
\hline \multicolumn{7}{|l|}{$\begin{array}{l}\text { Parent's education } \\
<\text { High school }\end{array}$} \\
\hline $\begin{array}{l}\text { < High school } \\
\text { High school graduate }\end{array}$ & $\begin{array}{r}0.07 \\
-\end{array}$ & $\begin{array}{l}1.07 \\
1.00\end{array}$ & 0.741 to 1.511 & 0.07 & $1 \cdot 07$ & 0.701 to 1.399 \\
\hline $\begin{array}{l}\text { H1gn school graauate } \\
>\text { High school }\end{array}$ & -0.007 & $\begin{array}{l}1.00 \\
0.99\end{array}$ & $\overline{0} .798$ to 1.385 & 0.02 & $\begin{array}{l}1.00 \\
1.02\end{array}$ & 0.881 to 1.574 \\
\hline \multicolumn{7}{|l|}{ Exercise (hours/week) } \\
\hline Low $(\leqslant 1)$ & - & 1.00 & & - & 1.00 & \\
\hline Medium (2-5) & -0.07 & 0.93 & 0.451 to 1.085 & $-1 \cdot 11$ & 0.33 & 0.667 to 1.235 \\
\hline High $(\geqslant 6)$ & 0.03 & 1.03 & 0.482 to 1.110 & $0 \cdot 14$ & 1.55 & 1.312 to 2.865 \\
\hline \multicolumn{7}{|l|}{ Employment (hours/week) } \\
\hline $\begin{array}{l}1-10 \\
\geqslant 11\end{array}$ & $\begin{array}{l}0.06 \\
0.05\end{array}$ & $\begin{array}{l}1.06 \\
1.05\end{array}$ & $\begin{array}{l}0.795 \text { to } 1.350 \\
0.712 \text { to } 1.477\end{array}$ & $\begin{array}{r}0 \cdot 18 \\
-0 \cdot 49\end{array}$ & $\begin{array}{l}1.20 \\
0.61\end{array}$ & $\begin{array}{l}0.992 \text { to } 1.739 \\
0.369 \text { to } 1.279\end{array}$ \\
\hline \multicolumn{7}{|c|}{ Alcohol use in past 30 days (days) } \\
\hline $\begin{array}{l}0 \\
1 \text { or } 2 \\
\geqslant 3\end{array}$ & $\begin{array}{r}- \\
-0 \cdot 10 \\
0.02\end{array}$ & $\begin{array}{l}1.00 \\
0.90 \\
1.02\end{array}$ & $\begin{array}{l}0.640 \text { to } 1.161 \\
0.699 \text { to } 1.279\end{array}$ & $\begin{array}{l}- \\
0.73 \\
0.26\end{array}$ & $\begin{array}{l}1 \cdot 00 \\
2 \cdot 07 \\
1 \cdot 30\end{array}$ & $\begin{array}{l}1.441 \text { to } 1.860 \\
0.845 \text { to } 1.825\end{array}$ \\
\hline \multicolumn{7}{|c|}{ Lifetime marijuana use (times used) } \\
\hline 0 & & 1.00 & & - & $1 \cdot 00$ & \\
\hline $\begin{array}{l}1-3 \\
\geq 6\end{array}$ & $0 \cdot 12$ & $1 \cdot 12$ & 0.857 to 1.527 & $0 \cdot 20$ & 1.22 & 0.756 to 1.691 \\
\hline \multicolumn{7}{|l|}{ Risk taking } \\
\hline $\begin{array}{l}\text { High (upper 25\%) } \\
\text { Medium }(50 \%)\end{array}$ & -0.45 & $\begin{array}{l}1.56 \\
1.00\end{array}$ & $1 \cdot 260$ to $2 \cdot 158$ & $-^{0.73}$ & $\begin{array}{l}2.07 \\
1.00\end{array}$ & 1.907 to 3.845 \\
\hline Low (lower $25 \%$ ) & -1.07 & 0.34 & 0.197 to 0.565 & -0.27 & 0.76 & 0.537 to 1.144 \\
\hline \multicolumn{7}{|l|}{ Cruising } \\
\hline $\begin{array}{l}\text { Several times weeks or less } \\
\text { School discipline problems }\end{array}$ & $0 \cdot 15$ & $1 \cdot 16$ & 0.659 to 1.210 & 0.53 & 1.69 & $1 \cdot 780$ to $2 \cdot 177$ \\
\hline $\begin{array}{l}\text { Yes/no } \\
\text { Anger }\end{array}$ & 0.41 & $1 \cdot 50$ & $1 \cdot 187$ to $2 \cdot 068$ & $0 \cdot 34$ & $1 \cdot 42$ & 0.836 to 1.553 \\
\hline High (upper 25\%) & 0.50 & 1.65 & $1 \cdot 277$ to $2 \cdot 134$ & 0.34 & 1.40 & 1.047 to 2.093 \\
\hline Medium $(50 \%)$ & - & 1.00 & & - & 1.00 & 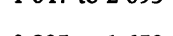 \\
\hline Low (lower 25\%) & 0.04 & $1 \cdot 04$ & 0.790 to 1.575 & -0.04 & $0 \cdot 96$ & 0.825 to 1.652 \\
\hline \multicolumn{7}{|l|}{ Parent supervision } \\
\hline Medium $(50 \%)$ & & 1.00 & 0.017 to 1.165 & -0.12 & $\begin{array}{l}0.88 \\
1.00\end{array}$ & 0.545 to 1.068 \\
\hline $\begin{array}{l}\text { Low (lower 25\%) } \\
\text { BMI }\end{array}$ & $-0 \cdot 10$ & 0.90 & 0.451 to 1.05 & $0 \cdot 11$ & $1 \cdot 11$ & 0.798 to 1.672 \\
\hline $\begin{array}{l}\text { High (upper 25\%) } \\
\text { Medium }(50 \%)\end{array}$ & -0.80 & $\begin{array}{l}1.08 \\
1.00\end{array}$ & 0.5108 to 9.463 & $\begin{array}{l}0 \cdot 20 \\
-\end{array}$ & $\begin{array}{l}1.22 \\
1.00\end{array}$ & 0.000 to 2.782 \\
\hline Low (lower $25 \%$ ) & -0.34 & $0 \cdot 78$ & 0.372 to 2.599 & 0.03 & 1.03 & 0.000 to 8.112 \\
\hline \multicolumn{7}{|c|}{ Pubertal status: girls (age menses started) } \\
\hline Early $(<11)$ & - & - & & $0 \cdot 77$ & $2 \cdot 15$ & $1 \cdot 777$ to $2 \cdot 8416$ \\
\hline On time $(11-13)$ & - & - & & - & $1 \cdot 00$ & \\
\hline \multirow{2}{*}{\multicolumn{7}{|c|}{ Pubertal status: boys (Tanner score) }} \\
\hline & & & & & & \\
\hline $\begin{array}{l}\text { Early }(\leqslant 6) \\
\text { On time }(7-8)\end{array}$ & $\begin{array}{r}0.07 \\
-\end{array}$ & $\begin{array}{l}1.07 \\
1.00\end{array}$ & 0.599 to 1.178 & $\overline{-}$ & - & \\
\hline $\begin{array}{l}\text { On time }(7-8) \\
\text { Late }(9+)\end{array}$ & 0.14 & $1 \cdot 15$ & 0.885 to 3.725 & $\overline{-}$ & $\overline{-}$ & \\
\hline \multicolumn{7}{|l|}{ Interactions } \\
\hline $\begin{array}{l}\text { Late pubertal status } \\
\times \text { high BMI }\end{array}$ & -0.002 & 0.99 & 0.299 to 2.113 & $2 \cdot 12$ & $5 \cdot 33$ & $\begin{array}{l}1 \cdot 2855 \text { to } \\
14 \cdot 080^{\dagger}\end{array}$ \\
\hline $\begin{array}{l}\text { Early pubertal status } \\
\times \text { low BMI }\end{array}$ & -0.08 & 0.92 & 0.235 to 1.208 & $0 \cdot 23$ & 1.26 & $0 \cdot 391$ to $2 \cdot 150$ \\
\hline $\begin{array}{l}\text { Late pubertal status } \times \text { low } \\
\text { BMI }\end{array}$ & $1 \cdot 13$ & 3.09 & 1.773 to 3.684 & $0 \cdot 36$ & $0 \cdot 70$ & $\begin{array}{l}0.000 \text { to } \\
3950 \cdot 00\end{array}$ \\
\hline $\begin{array}{l}\text { Early pubertal status } \times \\
\text { high BMI }\end{array}$ & 0.77 & $2 \cdot 16$ & 1.293 to 2.211 & $-1 \cdot 23$ & $0 \cdot 29$ & $\begin{array}{l}0.000 \text { to } \\
2482 \cdot 00\end{array}$ \\
\hline
\end{tabular}

${ }^{\star} \mathrm{CI}=$ Confidence interval. $†$ The wide $95 \% \mathrm{CI}$ for interaction coefficients is a function of large standard errors. might reflect differences in peer relationships, with girls tending to hang out with older adolescents. It may also represent rebellious behavior that tends to intensify at the apex of pubertal maturation. ${ }^{22} 23$ For most girls that intensification would occur in middle school, while, for boys, it could occur later, in high school.

For both girls and boys, high levels of expressed anger were generally associated with increased numbers of injuries whereas low levels of anger were associated with reduced numbers of injuries. Expressed anger may be a proxy for aggression. Boys and girls who report that they handle conflict through yelling, slamming doors, or even hitting, may be more reckless or volatile and thus more likely to engage in behaviors that can lead to injuries. The relationship between anger, aggression, and injury is one worth pursuing further.

Clearly, our findings suggest that adolescent injuries are related to a host of behaviors potentially linked to developmental change. This focus on development is unique in the area of research on adolescent injuries in which chronological age often serves as the indicator of developmental risk. Another strength of this study lies in its consideration of gender related biologic and behavioral risks for adolescent injury using a community sample. This is a departure from the reliance on data from emergency rooms, outpatient clinics, and hospitals.

One limitation of the study is the use of self reported injury information. Although adolescents in our study were asked to report only medically attended injuries, the relatively high proportions of students, males in particular, who reported one or more injuries, leads us to question whether reports were in fact restricted to those injuries for which they had received treatment by a physician or a nurse.

Another limitation concerns our inability to determine temporal relationships between behavior and injury. Behaviors were asked for different time periods (for example, 30 day alcohol use, lifetime marijuana use), whereas injuries were based on annual estimations. Because the reports of injuries were not specifically connected to the behaviors, we are unable to determine temporal sequencing and therefore cannot delineate causal relationships. In addition, we did not have information on the types of injuries experienced or on the circumstances under which the injuries occurred. Such data would have permitted an empirical examination of some of our hypothesized explanations for our gender related findings.

Lastly, differences between participants and non-participants could influence the generalizability of study findings. Respondents and non-respondents at the eighth grade recruitment were compared based on gender and race, the only demographic information made available to the researchers by the schools. There were no statistically significant differences in the distributions of gender and race by participation status. Without injury data, however, it is impossible to say whether injury rates for non-participants were less than, girls, while cruising was not a significant risk factor for boys. Because girls mature at an earlier age than boys, these gender differences 
the same as, or greater than the rates for study participants. If exposure specific injury rates for non-participants were higher than for participants, we would underestimate the 'true' relationships between risk taking, exercise, and other behaviors and injuries. If the rates of injury were lower for non-participants than for participants, then the effect of nonparticipation on the association between behaviors and injuries would be minimal. Without additional information on either behaviors or injury rates among nonparticipants, we can only speculate about the direction of selection bias.

\section{IMPLICATIONS FOR PREVENTION}

Despite these limitations, findings from this study suggest that parents, teachers, and physicians should increase their awareness of developmental risk factors for adolescent injuries. Rapid changes in body size and strength that occur during early adolescence can influence injury rates, particularly in middle school (grades 6-8). Middle school administrators need to be sensitive to the wide variation in physical development among students, especially among males, and structure athletic activities so as to minimize physical contact. Furthermore, physical development in adolescents is accompanied by social changes that may pose additional injury risks. Parents and physicians should recognize that, although many behaviors associated with injuries are performed by both genders, the sequencing of some risk taking behaviors differs for boys and girls, with girls initiating behaviors like substance use before high school. Finally, anger expression, not often examined in the context of adolescent injuries, should be studied further as a potential risk factor.

Data analyses for this paper were funded by the William $T$ Grant Foundation. The paper was presented at the 120th Annual American Public Health Association Meeting in Washington, DC, on 12 November 1992.
1 National Center for Health Statistics. Vital statistics of the United States, 1982. Mortality. Vol 1, part A. Washing ton, DC: US GPO, 1986. (US Department of Health and Human Services publication PHS 86-112.)

2 Gallagher SS, Finison K, Guyer B, Goodenough S. The incidence of injuries among 87,000 Massachusetts children and adolescents: results of the 1980-81 statewide childhood injury prevention surveillance system. Am $\mathcal{F}$ childhood injury prevention surve

3 American School Health Association, Association for the Advancement of Health Education, Society for Public Health Education Inc. The national adolescent student health survey: a report on the health of American's youth. Oakland, CA: Third Party Publishing, 1989.

4 Runyan CW, Gerken EA. Epidemiology and prevention of adolescent injury: a review and research agenda. $\mathcal{F} A M A$ 1989; 262: 2273-9.

5 Bass JL, Gallagher SS, Mebta KA. Injuries to adolescents and young adults. Pediatr Clin North Am 1985; 32: 31 -9.

6 Rivara FP, Muellar BA. The epidemiology and causes of childhood injuries. Fournal of Social Issues 1987; 43: 13-31.

7 Paulson JA. The epidemiology of injuries in adolescents. Pediatr Ann 1988; 17: 84-96.

8 Slap GB, Chaudhuri S, Vorters DF. Risk factors for injury during adolescence. $\mathcal{F}$ Adolesc Health 1991; 12: 263-8.

9 Hollinger PC. Self-destructiveness among the young: an epidemiologic study of violent deaths. Int $\mathcal{f}$ Soc Psychiatry 1981; 27: 277-83.

10 Alexander C, Ensminger M, Somerfield M, Kim Y, Johnson $\mathrm{K}$. Behavioral risk factors for injury among rural adolescents. Am $\mathcal{F}$ Epidemiol 1992; 136: 673-85.

11 Centers for Disease Control. Patterns of alcohol use among teenage drivers in fatal motor vehicle accidents - United States, 1977-1981. Morbidity and Mortality Weekly Report 1983; 32: 344-7.

12 Wagenaar AC. Alcohol, young drivers, and traffic accidents. Lexington, MA: Lexington Books, 1983.

13 Massachusetts Department of Public Health. The problem of sports and recreational injuries. SCIOO Reports 1983; 4: $1-4$.

14 Jacobsson B, Schelp L. One year incidence of occupational injuries among teenagers in a Swedish rural municipality. Scand F Soc Med 1988; 16: 21-5.

15 Baker S, O'Neill R, Karp R. Injury fact book. Lexington, MA: Mass Books, 1984.

16 Tanner JM. The course of children's growth. In: Hill JP, Sheldon J, eds. Readings in adolescent development and behavior. New Jersey: Prentice-Hall, 1971: 6-22.

17 Alexander C, Kim Y, Ensminger M, Johnson K, Smith J, Dolan L. A measure of risk taking for young adolescents: reliability and validity assessments. Fournal of Youth and Adolescence 1990; 19: 559-69.

18 Ensminger $M$, Brown $C$, Kellam $S$. Sex differences in antecedents of substance use among adolescents. Fournal of Social Issues 1982; 38: 23-42.

19 Rivara F. Epidemiology of childhood injuries: I. Review of current research and presentation of conceptual framework. Am ₹ Dis Child 1982; 136: 399-405.

20 Frome $E$. The analysis of rates using Poisson regression models. Biometrics 1983; 39: 665-74.

21 Peterson L, Farmer J, Mori L. Process analysis of injury situations: a complement to epidemiological methods. fournal of Social Issues 1987; 43: 33-44.

22 Steinberg L. Transformations in family relationships at puberty. Developmental Psychology 1981; 17: 833-40.

23 Papini DR, Sebby RA. Adolescent pubertal status and affective family relationships: a multivariate assessment. fournal of Youth and Adolescence 1987; 16: 1-15. 